\title{
Effect of Noise Sources on the Averaged PQRST Morphology
}

\author{
VU Medina $^{1}$, R González-Camarena ${ }^{2}$, JC Echeverría $^{1}$ \\ ${ }^{1}$ Electrical Engineering Department \\ ${ }^{2}$ Health Science Department \\ Universidad Autónoma Metropolitana-Izt, Mexico City, Mexico
}

\begin{abstract}
We analysed noise interference on the PQRST waveform of the electro- and magnetocardiogram as recovered by an average technique. Three signals (adult $A$, neonatal $N$, and fetal $F$ ) were corrupted with breathing, electromyographic and power-line noise at different levels of the $Q R S$ amplitude. Using the correlation coefficient $r$, averaged PQRST patterns from corrupted signals were compared with corresponding uncorrupted templates. Lower $r$ values were obtained at higher noise levels; this effect was more critical for the F signal. Noise sources caused different distortion; e.g. at 0.8 level and 50 averaged beats, $r$ remained high for breathing $(0.996 \mathrm{~A}, 0.995 \mathrm{~N}, 0.972 \mathrm{~F})$; lower for electromyography $(0.990 \mathrm{~A}, 0.972 \mathrm{~N}, 0.905 \mathrm{~F})$, and even lower for power-line $(0.931 \mathrm{~A}, 0.852 \mathrm{~N}, 0.574$ $F)$. Thus, to perform morphological analysis of the PQRST waveform the source, noise level and signal's nature should be considered for defining an optimal number of beats to be averaged.
\end{abstract}

\section{Introduction}

PQRST waveform analysis has proved to be a valuable approach for the identification of multiple cardiac diseases. Two main techniques electrocardiography (ECG) and magnetocardiography (MCG) are used as signal sources with this aim. However, in both techniques the waveform analysis could be precluded due to low signal/noise ratios $(\mathrm{S} / \mathrm{N})$ [1] that make the results inaccurate. In particular, fetal ECG recorded antenatally with electrodes placed on the abdominal surface represents a problem for the morphological analysis of fetal PQRST complexes [2]. Indeed, noise sources mainly from electromyographic activity, power-line, electrodes' interface, as well as maternal breathing and ECG are commonly found on the fetal ECG [2]. Starting with Hon and Lee in 1963 [3], time coherent averaging techniques have been used to reduce the impact of such interferences. Accordingly, different authors have averaged from 10 to more than 100 PQRST complexes of data derived from fetal ECG [4, 5, 6] and fetal MCG [7, 8, 9]. Since the magnitude and type of
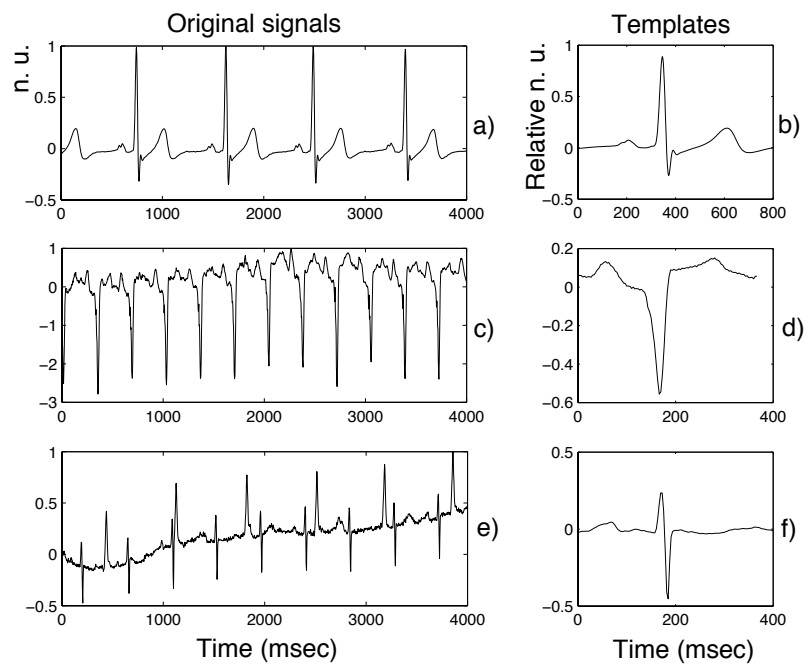

Figure 1. Segments of adult ECG (a), neonate ECG (c) and fetal MCG (e) as well as their corresponding templates (b, $\mathrm{d}$, f, respectively) obtained by averaging the entire signal.

noise sources can affect the waveforms at different levels, in this manuscript we analyse the noise interference caused by breathing, electromyographic and power-line sources on the morphologic characteristics of the PQRST complex as recovered by an average technique from 3 different signals.

\section{Method}

We used original 1 to 5 minute signals having high $\mathrm{S} / \mathrm{N}$ ratios $(>12 \mathrm{~dB})$, from an adult ECG $(\mathrm{A})$, a neonatal ECG $(\mathrm{N})$, and a fetal MCG $(\mathrm{F})$. By following standard procedures, signal A was obtained at $500 \mathrm{~Hz}$ from a resting 21 years old subject, whereas $\mathrm{N}$ was recorded at $300 \mathrm{~Hz}$ just after delivery. F signal was collected at $312.5 \mathrm{~Hz}$ from a 34 weeks gestation fetus as reported in [10]. This signal presented maternal complexes, so considered a noiseless equivalent of the abdominal ECG of pregnant woman. Figure 1 shows segments of the 3 original signals and their corresponding templates obtained by averaging the entire signal. We selected these signals to explore differences in morphology and heart frequency, as well as to obtain 
noiseless templates with a clear PQRST waveform.

Three sources of noise were added to each signal simulating fluctuations of the baseline given by maternal breathing (changing from 0.1 to $0.5 \mathrm{~Hz}$ ), electromyography (bandwidth below the Nyquist frequency), and power-line $(60 \mathrm{~Hz})$. Effect of each type of noise was independently assessed by increasing its levels at $0.1,0.4$ and 0.8 of the QRS complex amplitude. Original signals were beat-tobeat averaged to get their PQRST templates using the $\mathrm{R}$ wave as the time coherent alignment. For the corrupted signals, the PQRST patterns, obtained after averaging an increasing number of beats, were compared with the corresponding template by the correlation coefficient $r$, which was used as an index of agreement between corrupted and uncorrupted averaged PQRST complexes. For comparison purposes, the $r$ reached after averaging 50 complexes (R50), and the number of beats required to obtain an $r$ of 0.95 (N95) were derived from the behaviour of $r$ vs the increasing number of averaged complexes. Figure 2 illustrates the PQRST template and patterns of the F signal corrupted with electromyographic noise.
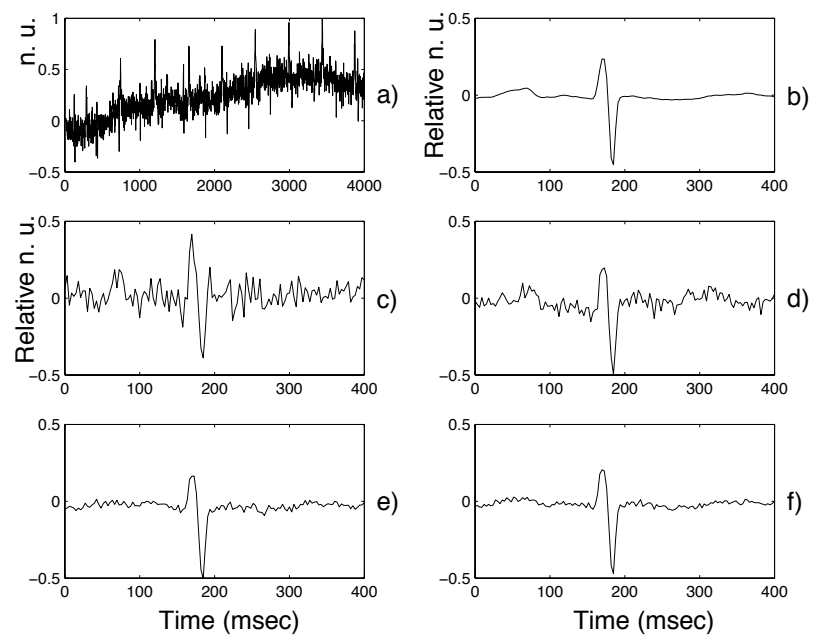

Figure 2. Segment of fetal MCG corrupted with electromyographic noise having 0.4 level of the QRS amplitude (a). The PQRST patterns obtained after averaging 2 (c), 10 (d), 50 (e), and 100 (f) beats are shown. To compare these patterns, the template obtained by averaging the entire noiseless original fetal MCG is also depicted (b). For this signal and noise level note the importance of averaging more than 50 beats. "n.u" stands for normalised units.

\section{Results}

For the three sources of noise (breathing, electromyography and power-line), the behaviour of $r$ increased tending to reach a plateau for all types and levels of noise

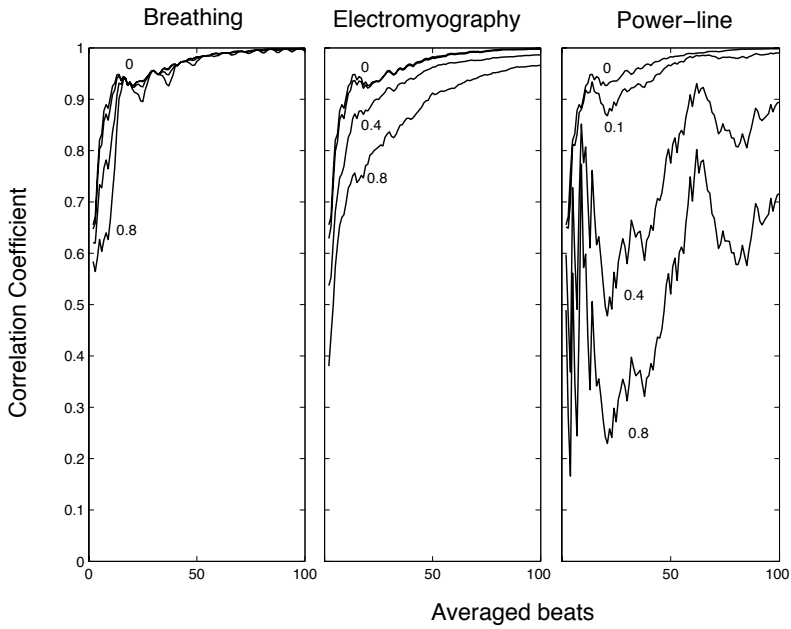

Figure 3. Correlation coefficient $(r)$ vs increasing number of averaged PQRST complexes for the fetal MCG signals corrupted with breathing, electromyographic and powerline interference at $0,0.1,0.4$ and 0.8 of the QRS complex amplitude.

(Figure 3). In fact (Table 1), the $r$ after averaging 50 complexes (R50) showed values above 0.95 at zero level of noise, being the $\mathrm{F}$ signal the one with the lowest value $(r=0.972)$ and $\mathrm{N}$ the one with the highest $(r=0.997)$. Accordingly, the number of beats required to obtain $r$ of 0.95 (N95) increased at higher levels of noise, being also the $\mathrm{F}$ signal the one requiring more beats to be averaged. Moreover, at 0.8 level of power-line interference it was not possible to reach $r=0.95$ after averaging the entire $\mathrm{F}$ signal. The correlation coefficients tend to be lower at higher levels of noise, but in particular this effect was more evident with the power-line and electromyographic interference for the $F$ signal (Figure 4). In addition, each source of noise caused different levels of distortion; e.g. at 0.8 level and 50 averaged beats the correlation coefficient for breathing remained high $(r=0.996,0.995,0.972$; for $\mathrm{A}, \mathrm{N}$ and $\mathrm{F}$ signals, respectively); for electromyography it was slightly lower ( $r=0.990,0.972,0.905 ; \mathrm{A}, \mathrm{N}$ and $\mathrm{F}$, respectively), and for power-line it was even lower $(r=0.931,0.852$, 0.574; A, N, F, respectively).

\section{Discussion}

Several authors have reported morphological measurements of the fetal ECG obtained by using average techniques without considering the type and level of noise [4-9]. As a fixed number of complexes is commonly used by these authors, an optimal number to be averaged has not been defined. Hence, the number has been selected from 10 to more than 100, which may result either in an inadequate precision when only few number of complexes 


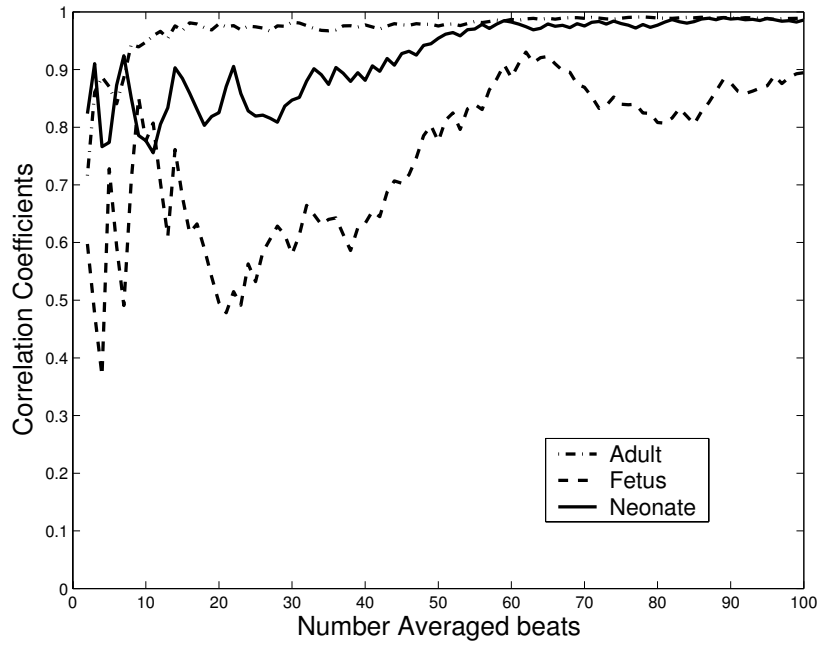

Figure 4. Correlation coefficient $(r)$ vs increasing number of averaged PQRST complexes for the adult ECG, neonatal ECG and fetal MCG signals corrupted with power-line noise at 0.4 level of the QRS amplitude.

are averaged, or in losing transitory information when several complexes are used. According to our results, we believe that such number cannot be predetermined as it would clearly depend on the level and type of noise interference, but also, importantly, on the nature of the signal. Clearly, the number of averaged beats necessary for getting a trustful PQRST waveform have to be increased at higher noise levels as already reported [3]. Regarding the noise sources, the effect on the PQRST waveform was different for each type of noise, despite reaching high $r$ values given a particular number of averaged beats. This indicates that a reliable waveform can be obtained after averaging different number of beats, which would depend on the type of noise. For example, concerning the $\mathrm{F}$ signal corrupted with 0.4 noise level, 28 averaged beats were required to obtain a presumed suitable morphology $\left(r^{2}>0.9\right)$ with respect to breathing, whereas 111 beats were needed for the power-line interference. The nature of the signal should also be considered as the $\mathrm{F}$ signal required more beats to be averaged. This may be related to the $\mathrm{F}$ signal distinctive features such as the $\mathrm{S} / \mathrm{N}$ ratio and its energy content above $30 \mathrm{~Hz}$, which is higher than the one found in adults $[11,12]$.

Finally, given the typical behaviour of $r$ (see figure 3-4), the evolution of a particular morphological measurement (for instance the PR interval) vs an increasing number of averaged complexes, could also show a similar asymptotical behaviour so becoming a convenient approach to derive the optimal number of complexes to be averaged for each particular signal.
Table 1. Effect of interference on signals' PQRST waveform, according to the noise type and noise level, evaluated by $r$ after averaging 50 beats (R50), and by the number of beats required for $r=0.95$ (N95). EMG = electromyography; "-" undetermined number of beats, because $r$ after averaging entire signal still $<0.95$

\begin{tabular}{|c|c|c|c|c|c|}
\hline & Noise Level & 0 & 0.1 & 0.4 & 0.8 \\
\hline \multicolumn{6}{|l|}{ Noise Type } \\
\hline \multicolumn{6}{|c|}{ Adult } \\
\hline \multirow[t]{2}{*}{ Breathing } & R50 & .996 & .996 & .996 & .996 \\
\hline & N95 & 1 & 1 & 1 & 5 \\
\hline \multirow[t]{2}{*}{ EMG } & R50 & .996 & .996 & .995 & .990 \\
\hline & N95 & 1 & 1 & 1 & 5 \\
\hline \multirow[t]{3}{*}{ Power-line } & R50 & .996 & .994 & .970 & .931 \\
\hline & N95 & 1 & 1 & 10 & 57 \\
\hline & \multicolumn{5}{|c|}{ Neonatal } \\
\hline \multirow[t]{2}{*}{ Breathing } & R50 & .997 & .997 & .997 & .995 \\
\hline & N95 & 1 & 1 & 1 & 1 \\
\hline \multirow[t]{2}{*}{ EMG } & R50 & .997 & .996 & .993 & .972 \\
\hline & N95 & 1 & 1 & 4 & 15 \\
\hline \multirow[t]{3}{*}{ Power-line } & R50 & .997 & .994 & .961 & .852 \\
\hline & N95 & 1 & 2 & 49 & 81 \\
\hline & & & & & \\
\hline \multirow[t]{2}{*}{ Breathing } & R50 & .972 & .972 & .972 & .972 \\
\hline & N95 & 28 & 28 & 28 & 28 \\
\hline \multirow[t]{2}{*}{ EMG } & R50 & .972 & .970 & .961 & .905 \\
\hline & N95 & 28 & 28 & 42 & 84 \\
\hline \multirow[t]{2}{*}{ Power-line } & $\mathrm{R} 50$ & .972 & .968 & .812 & .574 \\
\hline & N95 & 28 & 45 & 111 & - \\
\hline
\end{tabular}

\section{Conclusions}

Our results suggest that to perform morphological analysis of the PQRST waveform, the type and level of noise so as the nature of the signal should be considered for defining an optimal number of beats to be averaged. Moreover, the power-line noise, owing to its deterministic nature and frequency, seems to cause the most detrimental effect on the signals, in particular the fetal ones. Attempts to avoid this type of noise become convenient, particularly, when a fetal ECG morphological analysis is required.

\section{Acknowledgements}

The support of Dr. H. Preissl and Dr. D Gutierrez for providing fetal magnetocardiographic data, and Dr. C Vargas for the access to neonatal data collected at CIMIGen is gratefully acknowledged. 


\section{References}

[1] Stinstra J, Peters M. The influence of fetoabdominal tissues on fetal ECGs and MCGs. Arch Physiol Biochem 2002; 110:165-76.

[2] van Wijngaarden WJ, James DK, Symods EM. The fetal electrocardiogram. Baillieres Clin Obstet Gynaecol 1996; 10:273-94.

[3] Hon H, Lee S. Noise reduction in fetal electrocardiography. Am J Obstet Gynecol 1963;87:1086-96.

[4] Abboud S, Barkal G, Mashiach S, Sadeh D. Quantification of the fetal electrocardiogram using averaging technique. Comput Biol Med 1990;20:147-55.

[5] Huang XB, Crowe J, Herbert J, Woolfson M. A windows application for real-time fetal ECG analysis. Comput Biomed Res 1994;27:419-33.

[6] Brambati B, Pardi G. The intraventricular conduction time of fetal heart in uncomplicated pregnancies. Brit J Obstet Gynaecol 1980;87:941-8.

[7] Quinn A, Weir A, Shahani U, Bain R, Maas P, Donaldson G. Antenatal fetal magnetocardiography: a new method for fetal surveillance ? Brit J Obstet Gynaecol 1994;101:866870.

[8] Leuthold A, Wakai R, Martin C. Noninvasive in utero assessment of $\mathrm{PR}$ and QRS intervals from the fetal magnetocardiogram. Early Hum Dev 1999;54:235-43.

[9] Stinstra J, Golbach E, van Leeuwen P, Lange S, Menendez T, Moshage W, Schleussner E, Kaehler C, Horigome H, Shigemitsu S, Peters M. Multicentre study of fetal cardiac time intervals using magnetocardiography. Brit J Obstet Gynaecol 2002;109:1235-43.

[10] Guitierrez D, Nehorai A, McKenzie D, Eswaran H, Lowery CL, Preissl H. On-line fetal heart rate monitoring using SQUID sensor arrays. In Proceedings of the 14th Biennial BIOMAG Conference. Boston Massachusetts, USA, 2004; 315-6.

[11] Abboud S, Sadeh D. Spectral analysis of the fetal electrocardiogram. Comput Biol Med 1989;19:409-15.

[12] Piéri JF, Crowe JA, Hayes-Gill BR, Spencer CJ, Bhogal $\mathrm{K}$, James DK. Compact long-term recorder for the transabdominal foetal and maternal electrocardiogram. Med Biol Eng Comput 2001;39:118-25.

Address for correspondence:

R González-Camarena

UAM-I, Ingeniería Eléctrica

San Rafael Atlixco 186, 09340, México DF, México

rgc@xanum.uam.mx 